\title{
Stability Analysis of Second Order Delay Difference Equations
}

\author{
By \\ Hongshan REN \\ Heilongjiang University, P.R. China
}

\begin{abstract}
In this paper, we will give a necessary and sufficient condition for the zero solution of second order delay difference equations of the form

$$
x_{n+2}-a x_{n}+b x_{n-k}=0, \quad n=0,1,2, \ldots,
$$

to be asymptotically stable, which is easy to verify and to apply, where $a$ and $b$ are non-zero real constants, $k$ is a positive integer.

Key Words and Phrases. Delay difference equation, Asymptotic stability, Characteristic equation.

2000 Mathematics Subject Classification Numbers. 39A10, 39A11.
\end{abstract}

\section{Introduction}

In [1] Kuruklis established a necessary and sufficient condition for the asymptotic stability of first order delay difference equations of the form

$$
x_{n+1}-a x_{n}+b x_{n-k}=0, \quad n=0,1,2, \ldots,
$$

where $a$ and $b$ are constants, $k$ is a positive integer. In [2] Dannan discussed the asymptotic stability of a higher order delay difference equation of the form

$$
x(n+k)+a x(n)+b x(n-l)=0, \quad n=0,1,2, \ldots,
$$

where $a$ and $b$ are constants, $k$ and $l$ are positive integers. It appears, however, that there are several ambiguities in its main results and their proofs; more precisely, Lemma 6 ([2]) plays an essential role in the analysis of the characteristic roots (Lemmas 7 and 8), but its proof seems to be incomplete. In this paper, we will give an explicit criterion of the asymptotic stability for the case of second order $(k=2$ in $(*))$, that is, establish a necessary and sufficient condition for the asymptotic stability of delay difference equations of the form

$$
x_{n+2}-a x_{n}+b x_{n-k}=0, \quad n=0,1,2, \ldots,
$$

where $a$ and $b$ are constants, $k$ is a positive integer. Our criterion is described explicitly in terms of the constants $a, b$ and $k$ compared with the conditions 
due to [2]. Set $b(a, \theta)=\sqrt{a^{2}-2 a \cos 2 \theta+1}$, and for $x \in \boldsymbol{R}$ denote by $[x]$ the largest integer which does not exceed $x$. Then our main result is the following.

Theorem 1.1. Let $a, b$ be nonzero real constants, $k$ a positive integer.

(a) In the case $k$ is odd, the zero solution of Equation (1.1) is asymptotically stable, if and only if $0<a<1,|b|<1-a$, or $-1<a<0,|b|<b\left(a, \phi_{1}\right)$, where $\phi_{1}$ is the solution in the interval $((k+1) \pi / 2(k+2), \pi / 2)$ of the equation

$$
\sin (k+2) \theta / \sin k \theta=a \text {. }
$$

(b) In the case $k$ is even, the zero solution of Equation (1.1) is asymptotically stable, if and only if $0<a<(k+2) / k, a-1<b<b\left(a, \phi_{0}\right)$, or $-(k+2) / k<a<0,|a|-1<(-1)^{m} b<b\left(a, \phi_{2}\right)$, where $m=k / 2-2[k / 4]+1$, and $\phi_{0}$ and $\phi_{2}$ are the solutions of Equation (1.2) in $(0, \pi /(k+2))$ and $(k \pi / 2(k+2), \pi / 2)$, respectively.

Example 1.1. Consider the following equation

$$
x_{n+2}+\frac{1}{2} x_{n}+b x_{n-1}=0, \quad n=0,1,2, \ldots
$$

By Theorem 1.1(a), the zero solution of Equation (1.3) is asymptotically stable, if and only if $|b|<\sqrt{2} / 2$ holds, since $k=1, a=-1 / 2$ and $\phi_{1}=(1 / 2) \arccos (-3 / 4)$, and hence $b\left(-1 / 2, \phi_{1}\right)=\sqrt{2} / 2$.

Example 1.2. Consider the following equations

$$
\begin{array}{ll}
x_{n+2}-x_{n}+b x_{n-4}=0, & n=0,1,2, \ldots, \\
x_{n+2}+x_{n}+b x_{n-2}=0, & n=0,1,2, \ldots,
\end{array}
$$

and

$$
x_{n+2}+x_{n}+b x_{n-4}=0, \quad n=0,1,2, \ldots
$$

By Theorem 1.1(b), the zero solution of Equation (1.4) is asymptotically stable, if and only if $0<b<(\sqrt{5}-1) / 2$ holds, since $k=4, a=1$ and $\phi=$ $(1 / 2) \arccos ((1+\sqrt{5}) / 4)$, and hence $b\left(1, \phi_{0}\right)=(\sqrt{5}-1) / 2$; the zero solution of Equation (1.5) is asymptotically stable, if and only if $0<b<1$ holds, since $k=2, a=-1$ and $\phi_{2}=\pi / 3$, and hence $m=2$ and $b\left(-1, \phi_{2}\right)=1$; the zero solution of Equation (1.6) is asymptotically stable, if and only if $-(\sqrt{5}-1) / 2<$ $b<0$ holds, since $k=4, a=-1$ and $\phi_{2}=(1 / 2) \arccos (-((1+\sqrt{5}) / 4)$, and hence $m=1$ and $b\left(-1, \phi_{2}\right)=(\sqrt{5}-1) / 2$.

Remark 1.1. In this paper, we always assume that $a b \neq 0$, because under the condition $a b=0$ the result on stability of Equation (1.1) is well known. 


\section{Preparatory knowledge}

In this section, we introduce some notations and lemmas. Consider the equation

$$
\mu^{k+2}-\mu^{k}+c=0
$$

and the equation

$$
\mu^{k+2}+\mu^{k}+c=0,
$$

where $k$ is a positive integer, $c$ is a real number. Let

$$
\begin{gathered}
\alpha=\sqrt{\frac{k}{k+2}}, \\
\beta=\frac{2}{k+2}\left(\frac{k}{k+2}\right)^{k / 2} .
\end{gathered}
$$

Lemma 2.1. Let $k$ be a positive integer, $c$ be a nonzero real number.

(a) Assume that $k$ is odd. If $c>0$, then Equation (2.1) has exactly one negative root, denoted by $\mu_{1}(c)$, which belongs to $(-\infty,-1)$, is strictly decreasing for $c>0$ and satisfies $\mu_{1}\left(0^{+}\right)=-1, \lim _{c \rightarrow+\infty} \mu_{1}(c)=-\infty$. Also, if $0<c<\beta$, then it has exactly two positive roots, denoted by $\mu_{2}(c)$ and $\mu_{3}(c)$ with $\mu_{2}(c)<\mu_{3}(c)$, which satisfy $0<\mu_{2}(c)<\alpha<\mu_{3}(c)<1$, and $\mu_{2}(c) \quad\left(\right.$ resp. $\left.\mu_{3}(c)\right)$ is strictly increasing (resp. decreasing) with $\mu_{2}\left(0^{+}\right)=0, \mu_{2}\left(\beta^{-}\right)=\mu_{3}\left(\beta^{-}\right)=\alpha$ and $\mu_{3}\left(0^{+}\right)=1$; if $c=\beta$, then $\alpha$ is a double root; if $c>\beta$, then Equation (2.1) has no positive roots. If $c<0$, then $-\mu_{1}(-c)$ is the only positive root of Equation (2.1), which belongs to $(1,+\infty)$. Also, if $-\beta<c<0$, then $-\mu_{2}(-c)$ and $-\mu_{3}(-c)$ are the only two negative roots of Equation (2.1); if $c=-\beta$, then $-\alpha$ is a double root; if $c<-\beta$, then Equation (2.1) has no negative roots.

(b) Assume that $k$ is even. If $0<c<\beta$, then Equation (2.1) has exactly two positive roots and exactly two negative roots, denoted respectively by $\mu_{4}(c)$, $\mu_{5}(c),-\mu_{4}(c)$ and $-\mu_{5}(c)$ with $\mu_{4}(c)<\mu_{5}(c)$, which satisfy $0<\mu_{4}(c)<\alpha<$ $\mu_{5}(c)<1$, and $\mu_{4}(c)$ (resp. $\mu_{5}(c)$ ) is strictly increasing (resp. decreasing) in $0<c<\beta$ with $\mu_{4}\left(0^{+}\right)=0, \mu_{4}\left(\beta^{-}\right)=\mu_{5}\left(\beta^{-}\right)=\alpha, \mu_{5}\left(0^{+}\right)=1$; if $c=\beta$, then $-\alpha, \alpha$ are double roots; if $c>\beta$, then it has no real roots. If $c<0$, then Equation (2.1) has exactly one positive root and exactly one negative root, denoted respectively by $\mu_{6}(c)$ and $-\mu_{6}(c)$, which belong respectively to $(1,+\infty)$ and $(-\infty,-1)$, and $\mu_{6}(c)$ is strictly decreasing in $-\infty<c<0$ with $\lim _{c \rightarrow-\infty} \mu_{6}(c)=$ $+\infty, \mu_{6}\left(0^{-}\right)=1$.

Proof. Let $c(\mu)=\mu^{k}\left(1-\mu^{2}\right)$. Then $c^{\prime}(\mu)=\mu^{k-1}\left[k-(k+2) \mu^{2}\right]$. Therefore, the function $c(\mu)$ is strictly increasing in $[0, \alpha)$, reaches the maximum value $c(\alpha)=\beta$ at the point $\mu=\alpha$, strictly decreasing in $(\alpha,+\infty)$, and $c(0)=$ 
$c(1)=0, \lim _{\mu \rightarrow+\infty} c(\mu)=-\infty$; in the case that $k$ is odd, the function $c(\mu)$ is an odd function, in the case that $k$ is even, it is an even function. Thus, by the well known inverse function theorem, we complete the proof of this lemma.

Lemma 2.2. Let $k$ be a positive integer, $c$ be a nonzero real number.

(a) Assume that $k$ is odd. If $c>0$, then Equation (2.2) has no positive roots and has exactly one negative root, denoted by $\mu_{7}(c)$, which belongs to $(-\infty, 0)$, and is strictly decreasing in $0<c<+\infty$ with $\mu_{7}\left(0^{+}\right)=0$, $\lim _{c \rightarrow+\infty} \mu_{7}(c)=-\infty$. If $c<0$, then Equation (2.2) has no negative roots and $-\mu_{7}(-c)$ is the only one positive root.

(b) Assume that $k$ is even. If $c>0$, then Equation (2.2) has no real roots. If $c<0$, then Equation (2.2) has one positive root and one negative root, denoted respectively by $\mu_{8}(c)$ and $-\mu_{8}(c) ; \mu_{8}(c)$ belongs to $(0,+\infty)$ and is strictly decreasing in $-\infty<c<0$ with $\lim _{c \rightarrow-\infty} \mu_{8}(c)=+\infty, \mu_{8}\left(0^{-}\right)=0$.

The proof of Lemma 2.2 is similar to the one of Lemma 2.1, so we will omit.

Lemma 2.3. Let $k$ be a positive integer, $c$ be a nonzero real number.

(a) Assume that $k$ is odd. Then Equation (2.1) has no pure imaginary roots.

(b) Assume that $k \equiv 2(\bmod 4)$. If $c>0$, then Equation (2.1) has no pure imaginary roots; if $c<0$, then $\mu_{8}(c) i$ and $-\mu_{8}(c) i$ are the only two pure imaginary roots of Equation (2.1).

(c) Assume that $k \equiv 0(\bmod 4)$. If $c>0$, then $\mu_{8}(-c) i$ and $-\mu_{8}(-c) i$ are the only two pure imaginary roots of Equation (2.1); if $c<0$, Equation (2.1) has no pure imaginary roots.

Here $\mu_{8}(c)$ is the one in Lemma 2.2(b).

Proof. (a) Set $f(\mu):=\mu^{k+2}-\mu^{k}+c$. It is easy to see that in the case that $k$ is odd, $f(i y) \neq 0$, for $\forall y \in \boldsymbol{R}$, that is, Equation (2.1) has no pure imaginary roots.

(b) Let $\mu:=i y$, then in the case that $k=4 m+2, m=0,1,2, \ldots$, Equation (2.1) becomes $y^{4 m+4}+y^{4 m+2}+c=0$. Consequently, by Lemma 2.2(b), we obtain the conclusion of Part (b).

(c) Let $\mu:=i y$, then in the case that $k=4 m, m=1,2, \ldots$, Equation (2.1) becomes $y^{4 m+2}+y^{4 m}-c=0$. Consequently, by Lemma 2.2(b), we obtain the conclusion of Part (c).

The proof is complete.

Lemma 2.4. Let $k$ be a positive integer, $c$ be a nonzero real number.

(a) Assume that $k$ is odd. Then Equation (2.2) has no pure imaginary roots. 
(b) Assume that $k \equiv 2(\bmod 4)$. If $0<c<\beta$, then $\mu_{4}(c) i, \mu_{5}(c) i,-\mu_{4}(c) i$ and $-\mu_{5}(c) i$ are the only four pure imaginary roots of Equation (2.2); if $c=\beta$, then $-\alpha i$, $\alpha i$ are double roots; if $c>\beta$, then it has no pure imaginary roots. If $c<0, \mu_{6}(c) i$ and $-\mu_{6}(c) i$ are the only two pure imaginary roots.

(c) Assume that $k \equiv 0(\bmod 4)$. If $c>0$, then $-\mu_{6}(-c) i$ and $\mu_{6}(-c) i$ are the only two pure imaginary roots of Equation (2.2). If $-\beta<c<0$, then $-\mu_{4}(-c) i,-\mu_{5}(-c) i, \mu_{4}(-c) i$ and $\mu_{5}(-c) i$ are the only four pure imaginary roots; if $c=-\beta$, then $-\alpha i$, $\alpha i$ are double roots; if $c<-\beta$, then (2.2) has no pure imaginary roots.

Here $\mu_{j}(c)(j=3,4,5,6)$ are the ones in Lemma 2.1(b).

Proof. (a) Set $g(\mu):=\mu^{k+2}+\mu^{k}+c$. It is easy to see that in the case that $k$ is odd, $g(i y) \neq 0$, for $\forall y \in \boldsymbol{R}$, that is, Equation (2.2) has no pure imaginary roots.

(b) Let $\mu:=i y$, then in the case that $k=4 m+2, m=0,1,2, \ldots$, Equation (2.2) becomes $y^{4 m+4}-y^{4 m+2}+c=0$. Consequently, by Lemma 2.1(b), we obtain the conclusion of Part (b).

(c) Let $\mu:=i y$, then in the case that $k=4 m, m=1,2, \ldots$, Equation (2.1) becomes $y^{4 m+2}-y^{4 m}-c=0$. Consequently, by Lemma 2.1(b), we obtain the conclusion of Part (c).

The proof is complete.

Consider the following subsets of the interval $(0, \pi / 2)$ :

$$
\begin{aligned}
& E_{k}^{++}=\{0<\theta<\pi / 2 \mid \sin k \theta>0, \sin (k+2) \theta>0\}, \\
& E_{k}^{+-}=\{0<\theta<\pi / 2 \mid \sin k \theta>0, \sin (k+2) \theta<0\}, \\
& E_{k}^{-+}=\{0<\theta<\pi / 2 \mid \sin k \theta<0, \sin (k+2) \theta>0\}, \\
& E_{k}^{--}=\{0<\theta<\pi / 2 \mid \sin k \theta<0, \sin (k+2) \theta<0\} .
\end{aligned}
$$

Let $k$ be an odd positive integer. Then $\sin k \theta>0$ and $0<\theta<\pi / 2$ are equivalent to

$$
\frac{2 m \pi}{k}<\theta<\frac{(2 m+1) \pi}{k}, \quad m=0,1, \ldots,\left[\frac{k}{4}\right]-1, \text { or } \frac{(k-1) \pi}{2 k}<\theta<\frac{\pi}{2},
$$

when $k \equiv 1(\bmod 4)$, and also are equivalent to

$$
\frac{2 m \pi}{k}<\theta<\frac{(2 m+1) \pi}{k}, \quad m=0,1, \ldots,\left[\frac{k}{4}\right]
$$

when $k \equiv 3(\bmod 4)$. Similarly, $\sin (k+2) \theta>0$ and $0<\theta<\pi / 2$ are equivalent to 


$$
\frac{2 n \pi}{k+2}<\theta<\frac{(2 n+1) \pi}{k+2}, \quad n=0,1, \ldots,\left[\frac{k}{4}\right],
$$

when $k \equiv 1(\bmod 4)$, and also are equivalent to

$$
\frac{2 n \pi}{k+2}<\theta<\frac{(2 n+1) \pi}{k+2}, \quad n=0,1, \ldots,\left[\frac{k}{4}\right], \text { or } \frac{(k+1) \pi}{2(k+2)}<\theta<\frac{\pi}{2},
$$

when $k \equiv 3(\bmod 4)$. So it is easy to see that,

$$
E_{k}^{++}=\bigcup_{p=0}^{[k / 4]}\left(\frac{2 p \pi}{k}, \frac{(2 p+1) \pi}{k+2}\right)
$$

By a similar simple calculation one can readily verify the following lemma, its proof will be omitted.

Lemma 2.5. (i) Suppose that $k$ is an odd integer. Then

(a) $E_{k}^{++}=\bigcup_{p=0}^{[k / 4]}\left(\frac{2 p \pi}{k}, \frac{(2 p+1) \pi}{k+2}\right)$.

(b) $E_{1}^{--}=\varnothing$, and for $k>1$,

$$
E_{k}^{--}= \begin{cases}\bigcup_{p=0}^{[k / 4]-1}\left(\frac{(2 p+1) \pi}{k}, \frac{(2 p+2) \pi}{k+2}\right), & k \equiv 1(\bmod 4), \\ \bigcup_{p=0}^{[k / 4]}\left(\frac{(2 p+1) \pi}{k}, \frac{(2 p+2) \pi}{k+2}\right), & k \equiv 3(\bmod 4) .\end{cases}
$$

(c) $E_{1}^{+-}=(\pi / 3, \pi / 2)$, for $k>1$,

$$
E_{k}^{+-}= \begin{cases}\bigcup_{p=0}^{[k / 4]-1}\left(\frac{(2 p+1) \pi}{k+2}, \frac{(2 p+1) \pi}{k}\right) \cup\left(\frac{(k+1) \pi}{2(k+2)}, \frac{\pi}{2}\right), & k \equiv 1(\bmod 4), \\ \bigcup_{p=0}^{[k / 4]}\left(\frac{(2 p+1) \pi}{k+2}, \frac{(2 p+1) \pi}{k}\right), & k \equiv 3(\bmod 4) .\end{cases}
$$

(d) $E_{1}^{-+}=\varnothing, E_{3}^{-+}=(2 \pi / 5, \pi / 2)$, and for $k \neq 1,3$,

$$
E_{k}^{-+}= \begin{cases}\bigcup_{p=0}^{[k / 4]-1}\left(\frac{(2 p+2) \pi}{k+2}, \frac{(2 p+2) \pi}{k}\right), & k \equiv 1(\bmod 4), \\ \bigcup_{p=0}^{[k / 4]-1}\left(\frac{(2 p+2) \pi}{k+2}, \frac{(2 p+2) \pi}{k}\right) \cup\left(\frac{(k+1) \pi}{2(k+2)}, \frac{\pi}{2}\right), & k \equiv 3(\bmod 4) .\end{cases}
$$

(ii) Suppose that $k$ is an even integer. Then

(a)

$$
E_{k}^{++}= \begin{cases}\bigcup_{p=0}^{[k / 4]-1}\left(\frac{2 p \pi}{k}, \frac{(2 p+1) \pi}{k+2}\right), & k \equiv 0(\bmod 4), \\ \bigcup_{p=0}^{[k / 4]}\left(\frac{2 p \pi}{k}, \frac{(2 p+1) \pi}{k+2}\right), & k \equiv 2(\bmod 4) .\end{cases}
$$

(b) $E_{2}^{--}=\varnothing$, and for $k>2, E_{k}^{--}=\bigcup_{p=0}^{[k / 4]-1}\left(\frac{(2 p+1) \pi}{k}, \frac{(2 p+2) \pi}{k+2}\right)$, 
(c)

$$
E_{k}^{+-}= \begin{cases}\bigcup_{p=0}^{[k / 4]-1}\left(\frac{(2 p+1) \pi}{k+2}, \frac{(2 p+1) \pi}{k}\right), & k \equiv 0(\bmod 4), \\ \bigcup_{p=0}^{[k / 4]-1}\left(\frac{(2 p+1) \pi}{k+2}, \frac{(2 p+1) \pi}{k}\right) \cup\left(\frac{k \pi}{2(k+2)}, \frac{\pi}{2}\right), & k \equiv 2(\bmod 4) .\end{cases}
$$

(d) $E_{2}^{-+}=\varnothing$, and for $k>2$,

$$
E_{k}^{-+}= \begin{cases}\bigcup_{p=0}^{[k / 4]-2}\left(\frac{(2 p+2) \pi}{k+2}, \frac{(2 p+2) \pi}{k}\right) \cup\left(\frac{k \pi}{2(k+2)}, \frac{\pi}{2}\right), & k \equiv 0(\bmod 4), \\ \bigcup_{p=0}^{[k / 4]-1}\left(\frac{(2 p+2) \pi}{k+2}, \frac{(2 p+2) \pi}{k}\right), & k \equiv 2(\bmod 4) .\end{cases}
$$

Lemma 2.6. The absolute values of the roots of Equation (2.1) increase as $|c|$ increases except in the following cases:

(a) $k$ is odd. In this case, if $0<c<\beta$, then the largest positive root $\mu_{3}(c)$ of Equation (2.1), which lies in $(\alpha, 1)$, decreases as $c$ increases; if $-\beta<c<0$, then the smallest negative root $-\mu_{3}(-c)$ of Equation (2.1), which lies in $(-1,-\alpha)$, increases as c decreases.

(b) $k$ is even and $0<c<\beta$. In this case, the absolute values of both the smallest negative root $-\mu_{5}(c)$ and the largest positive root $\mu_{5}(c)$ of Equation (2.1), which lie respectively in $(-1,-\alpha)$ and $(\alpha, 1)$, decrease as $c$ increases.

Proof. By virtue of Lemma 2.1 and Lemma 2.3, this lemma is valid for both the real roots and the pure imaginary roots. Let $\mu=r(\cos \theta+i \sin \theta)$ be a root of Equation (2.1) which is neither real nor pure imaginary, namely $\theta \in(0,2 \pi) \backslash\{\pi / 2, \pi, 3 \pi / 2\}$. Substituting it into Equation (2.1), we have

$$
\begin{aligned}
& r^{k}\left[\cos k \theta-r^{2} \cos (k+2) \theta\right]=c, \\
& r^{k}\left[\sin k \theta-r^{2} \sin (k+2) \theta\right]=0 .
\end{aligned}
$$

In view of $2 \theta \neq \pi, 2 \pi, 3 \pi$, Equation (2.6) implies

$$
r^{2}=\frac{\sin k \theta}{\sin (k+2) \theta}
$$

Since the non-real roots of Equation (2.1) are the conjugate pairs and the graph of the function $\sin k \theta / \sin (k+2) \theta$ is symmetric with respect to the line $\theta=\pi / 2$, we may assume that $\theta \in(0, \pi / 2)$. Thus, by (2.7) it suffices to restrict $\theta$ within $E_{k}^{++} \cup E_{k}^{--}$. Note that Equations (2.5) and (2.7) yield

$$
c=\frac{r^{k} \sin 2 \theta}{\sin (k+2) \theta} \text {. }
$$

From (2.7) and (2.8), it follows that

$$
\frac{d r}{d \theta}=\frac{G(\theta)}{2 r \sin ^{2}(k+2) \theta}
$$


and

$$
\frac{d c}{d \theta}=k r^{k-1} \frac{d r}{d \theta} \frac{\sin 2 \theta}{\sin (k+2) \theta}+r^{k} \frac{H(\theta)}{\sin ^{2}(k+2) \theta}
$$

where

$$
G(\theta)=k \cos k \theta \sin (k+2) \theta-(k+2) \cos (k+2) \theta \sin k \theta
$$

and

$$
H(\theta)=2 \cos 2 \theta \sin (k+2) \theta-(k+2) \cos (k+2) \theta \sin 2 \theta .
$$

Hence

$$
\frac{d c}{d \theta}=r^{k} \frac{K(\theta)}{2 \sin k \theta \sin ^{2}(k+2) \theta},
$$

where

$$
K(\theta)=k \sin 2 \theta G(\theta)+2 \sin k \theta H(\theta) .
$$

Therefore if $d c / d \theta \neq 0$, we have

$$
\frac{d r}{d c}=\frac{\sin k \theta G(\theta)}{r^{k+1} K(\theta)}
$$

Notice that both $G(\theta)$ and $K(\theta)$ are strictly increasing in $E_{k}^{++} \cup E_{k}^{--}$because $G^{\prime}(\theta)=4(k+1) \sin k \theta \sin (k+2) \theta \quad$ and $\quad K^{\prime}(\theta)=8 k(k+2) \sin 2 \theta \sin k \theta \sin$. $(k+2) \theta$.

Next, consider the two cases.

Case 1: $k$ is odd. Note that $G(2 p \pi / k)=k \sin (4 p \pi / k) \geq 0$ for $p=$ $0,1, \ldots,[k / 4]$ and $G((2 p+1) \pi / k)=k \sin (4 p+2) \pi / k>0$ for $p=0,1, \ldots, i_{k}$, where $i_{k}=[k / 4]-1$ when $k \equiv 1(\bmod 4)$ and $i_{k}=[k / 4]$ when $k \equiv 3(\bmod 4)$. Lemma 2.5(i)(a) and (b), together with the monotonicity of $G(\theta)$, implies $G(\theta)>0$ for $\theta \in E_{k}^{++} \cup E_{k}^{--}$. Similarly, $K(\theta)>0$ for $\theta \in E_{k}^{++} \cup E_{k}^{--}$because $K(2 p \pi / k)=k^{2} \sin ^{2}(4 p \pi / k) \geq 0$ for $p=0,1, \ldots,[k / 4]$ and $K((2 p+1) \pi / k)=$ $k^{2} \sin ^{2}((4 p+2) \pi / k)>0$ for $p=0,1, \ldots, i_{k}$. In view of $(2.8) c$ has the same sign as $\sin k \theta$ in $E_{k}^{++} \cup E_{k}^{--}$, and it therefore follows from (2.10) that $c(d r / d c)>0$ for $\theta \in E_{k}^{++} \cup E_{k}^{--}$.

Case 2: $k$ is even. Note that $G(2 p \pi / k) \geq 0$, and $K(2 p \pi / k) \geq 0$ for $p=0,1, \ldots, j_{k}$, where $j_{k}=[k / 4]-1$ when $k \equiv 0(\bmod 4)$ and $[k / 4]$ when $k \equiv 2(\bmod 4)$, and $G((2 p+1) \pi / k)>0$ and $K((2 p+1) \pi / k)>0$ for $p=$ $0,1, \ldots,[k / 4]-1$ (see Lemma $2.5(\mathrm{ii})(\mathrm{a})$ and $(\mathrm{b})$ ). By the same reasoning as 
Case 1, we obtain that $c(d r / d c)>0$ for $\theta \in E_{k}^{++} \cup E_{k}^{--}$; consequently, the conclusion holds.

The proof is complete.

Lemma 2.7. The absolute values of the roots of Equation (2.2) increase as $|c|$ increases except in the following cases:

(a) $k \equiv 2$ (mod) 4 and $0<c<\beta$. In this case, the absolute values of the pair of pure imaginary roots $\pm \mu_{5}(c) i$ of Equation (2.2) (see Lemma 2.4) decrease as c increases.

(b) $k \equiv 0$ (mod) 4 and $-\beta<c<0$. In this case, the absolute values of the pair of pure imaginary roots $\pm \mu_{5}(-c) i$ of Equation (2.2) (see Lemma 2.4) decrease as c decreases.

Proof. By virtue of Lemma 2.2 and Lemma 2.4, this lemma is valid for both the real roots and the pure imaginary roots. Let $\mu=r(\cos \theta+i \sin \theta)$ be a root of Equation (2.2) with $\theta \in(0,2 \pi) \backslash\{\pi / 2, \pi, 3 \pi / 2\}$. Then we obtain from Equation (2.2)

$$
\begin{gathered}
r^{k}\left[\cos k \theta+r^{2} \cos (k+2) \theta\right]=-c, \\
r^{k}\left[\sin k \theta+r^{2} \sin (k+2) \theta\right]=0,
\end{gathered}
$$

and instead of (2.7), (2.8), we have

$$
r^{2}=-\frac{\sin k \theta}{\sin (k+2) \theta}, \quad c=-\frac{r^{k} \sin 2 \theta}{\sin (k+2) \theta},
$$

respectively. By the same reasoning as the proof of Lemma 2.6, it suffices to restrict $\theta$ within $E_{k}^{+-} \cup E_{k}^{-+}$, and a similar calculation yields that if $d c / d \theta \neq 0$,

$$
\frac{d r}{d c}=-\frac{\sin k \theta G(\theta)}{r^{k+1} K(\theta)},
$$

where $G(\theta)$ and $K(\theta)$ are the functions in the proof of Lemma 2.6. Notice that both $G(\theta)$ and $K(\theta)$ are strictly decreasing in $E_{k}^{+-} \cup E_{k}^{-+}$. Consider the two cases.

Case 1: $k$ is odd. Note that $G(\pi / 2)=0, G((2 p+1) \pi / k)>0$ for $p=$ $0,1, \ldots, i_{k}, i_{k}$ being the one in the proof of Lemma 2.6 , and $G((2 p+2) \pi / k)>0$ for $p=0,1, \ldots,[k / 4]-1$. Then Lemma $2.5(\mathrm{i})(\mathrm{c})$ and $(\mathrm{d})$, together with the monotonicity of $G(\theta)$, implies $G(\theta)>0$ for $\theta \in E_{k}^{+-} \cup E_{k}^{-+}$. Similarly, $K(\theta)>0$ for $\theta \in E_{k}^{+-} \cup E_{k}^{-+}$because of $K(\pi / 2)=0, K((2 p+1) \pi / k)>0$ for $p=0,1, \ldots, i_{k}$ and $K((2 p+2) \pi / k)>0$ for $p=0,1, \ldots,[k / 4]-1$. In view of (2.13) $c$ has the opposite sign as $\sin k \theta$ in $E_{k}^{+-} \cup E_{k}^{-+}$, and it follows from (2.14) that $c(d r / d c)>0$ for $\theta \in E_{k}^{+-} \cup E_{k}^{-+}$. 
Case 2: $\quad k$ is even. Note that $G((2 p+1) \pi / k)>0$ and $K((2 p+1) \pi / k)>0$ for $p=0,1, \ldots,[k / 4]-1$, and $G((2 p+2) \pi / k) \geq 0$ and $K((2 p+2) \pi / k) \geq 0$ for $p=0,1, \ldots, j_{k}^{\prime}$, where $j_{k}^{\prime}=[k / 4]-2$ when $k \equiv 0(\bmod 4)$ and $[k / 4]-1$ when $k \equiv 2(\bmod 4)($ see Lemma $2.5(\mathrm{ii})(\mathrm{c})$ and $(\mathrm{d}))$. In the same way as Case 1, we obtain that $c(d r / d c)>0$ for $\theta \in E_{k}^{+-} \cup E_{k}^{-+}$. This completes the proof.

Lemma 2.8. For $r_{0} \geq 0$ and $\theta \in[0,2 \pi)$ then the following holds:

(a) If $r_{0} e^{i \theta}$ be a root of Equation (2.1), then

$$
|c|=r_{0}^{k} \sqrt{r_{0}^{4}-2 r_{0}^{2} \cos 2 \theta+1} .
$$

(b) If $r_{0} e^{i \theta}$ be a root of Equation (2.2), then

$$
|c|=r_{0}^{k} \sqrt{r_{0}^{4}+2 r_{0}^{2} \cos 2 \theta+1} .
$$

Proof. From (2.1) it immediately follows that

$$
|c|=\left|r_{0}^{k+2} e^{i(k+2) \theta}-r_{0}^{k} e^{i k \theta}\right|=r_{0}^{k}\left|r_{0}^{2} e^{i 2 \theta}-1\right|,
$$

which yields (a). The proof of (b) is similar. The proof is complete.

Remark 2.1. In view of (2.15), the minimum of $|c|$ such that Equation (2.1) has a root on the circle $|\mu|=r_{0}$ is $r_{0}^{k}\left|r_{0}^{2}-1\right|$, and is attained when the root is $r_{0}$ or $-r_{0}$. Note that the roots of Equation (2.1) with $c=0$ consist of 0 (multiplicity $k$ ) and \pm 1 (simple). Therefore, the continuous dependence on $c$ of the roots of Equation (2.1), combined with Lemmas 2.1 and 2.3, implies that if $|c| \leq r_{0}^{k}\left|r_{0}^{2}-1\right|$, then every non-real root $\mu$ of Equation (2.1) satisfies $|\mu|<r_{0}$. By a similar argument one can see that if $|c| \leq r_{0}^{k}\left|r_{0}^{2}-1\right|$, then every non-pure imaginary root $\mu$ of Equation (2.2) satisfies $|\mu|<r_{0}$.

Remark 2.2. If $a>0$ (resp. $a<0$ ) and Equation (2.1) (resp. Equation (2.2)) has a root $r e^{i \theta}$ on the circle $|\mu|=1 / \sqrt{|a|}$, then

$$
|c|=\frac{\sqrt{a^{2}-2 a \cos 2 \theta+1}}{|a|^{(k+2) / 2}}=: b_{*}(a, \theta) .
$$

Let $S(\theta):=\sin (k+2) \theta / \sin k \theta$ and consider Equation (1.2).

Lemma 2.9. If $0<a<1 / \alpha^{2}$, then the minimum of $c>0$ such that Equation (2.1) has a root, which is neither real nor pure imaginary, on the circle $|\mu|=$ $1 / \sqrt{a}$ is given by $b_{*}\left(a, \phi_{0}\right)$, where $\phi_{0}$ is the solution of $(1.2)$ in the interval $(0, \pi /(k+2))$.

Proof. Obviously, the arguments of the roots of Equation (2.1) on the circle $|\mu|=1 / \sqrt{a}$, except for the real roots and pure imaginary ones, are precisely the solutions of (1.2). In virtue of Remark 2.2 the required minimum of $c>0$ is given by $(2.17)$ with $\theta \in[0,2 \pi)$ which is the minimum solution 
of (1.2). Notice that $S\left(0^{+}\right)=1 / \alpha^{2}$ and $S(\pi /(k+2))=0$. Since by (2.7) and (2.9) $S(\theta)$ is strictly decreasing in $E_{k}^{++} \cup E_{k}^{--}$and hence in $(0, \pi /(k+2))$, the assumption implies that (1.2) has a unique solution in this interval. Denote the solution by $\phi_{0}$. Clearly $\phi_{0}$ is the minimum solution of (1.2). The proof is complete.

Let $c_{*}\left(\right.$ resp. $\left.c^{*}\right)$ be maximum of $c<0$ (resp. minimum of $c>0$ ) such that Equation (2.2) has a root, which is neither real nor pure imaginary, on the circle $|\mu|=1 / \sqrt{|a|}$.

Lemma 2.10. (a) If $k$ is odd and $-1<a<0$, then $c_{*}=-b_{*}\left(a, \phi_{1}\right)$ and $c^{*}=b_{*}\left(a, \phi_{1}\right)$, where $\phi_{1}$ is the solution of $(1.2)$ in the interval $((k+1) \pi /$ $(2(k+2)), \pi / 2)$.

(b) If $k$ is even and $-1 / \alpha^{2}<a<0$, then $c_{*}=-b_{*}\left(a, \phi_{2}\right)$ and $c^{*}=$ $b_{*}\left(a, \phi_{2}\right)$, where $\phi_{2}$ is the solution of (1.2) in the interval $(k \pi /(2(k+2)), \pi / 2)$.

Proof. By the same reasoning as the proof of Lemma 2.9, together with Remark 2.2 and the fact $a<0,\left|c_{*}\right|$ and $c^{*}$ are given by (2.17) with $\theta \in[0,2 \pi)$ which is the solution of (1.2) closest to $\pi / 2$. Moreover, since $S(\pi / 2-\psi)=$ $S(\pi / 2+\psi)$ for $\psi \in[0, \pi / 2)$, it is sufficient to restrict $\theta$ within $[0, \pi / 2)$.

(a) Notice that $S(\theta)$ is strictly decreasing in $E_{k}^{+-} \cup E_{k}^{-+}$, and hence so is in $((k+1) \pi /(2(k+2)), \pi / 2)$. Since $S(\pi / 2-0)=-1$ and $S((k+1) \pi /(2(k+2)))$ $=0$, it thus follows from $-1<a<0$ that (1.2) has a unique solution, denoted $\phi_{1}$, in this interval. Obviously $\phi_{1}$ is the solution of (1.2) closest to $\pi / 2$. This proves (a).

(b) Similarly, by the monotonicity of $S(\theta)$ in $(k \pi /(2(k+2)), \pi / 2)$ and the fact $S(\pi / 2-0)=-1 / \alpha^{2}$ and $S(k \pi /(2(k+2)))=0$, the assumption $-1 / \alpha^{2}<$ $a<0$ implies that (1.2) has a unique solution, denoted $\phi_{2}$, in this interval. $\phi_{2}$ is the solution of $(1.2)$ closest to $\pi / 2$, which proves (b).

The proof is complete.

\section{Proof of Theorem 1.1}

In this section, we give a proof of Theorem 1.1. The characteristic equation of Equation (1.1) is as follows.

$$
\lambda^{k+2}-a \lambda^{k}+b=0 .
$$

By setting $\lambda=\sqrt{|a|} \mu$, Equation (3.1) becomes Equations (2.1) and (2.2) with

$$
c=\frac{b}{|a|^{(k+2) / 2}}
$$

in the cases $a>0$ and $a<0$, respectively. 
It is well known that the zero solution of Equation (1.1) is asymptotically stable, if and only if all the roots of Equation (3.1) lie inside the unit open circular disk. Therefore, it follows that the zero solution of Equation (1.1) is asymptotically stable, if and only if either $a>0$ and all the roots of Equation (2.1) with (3.2) are inside the disk $|\mu|<1 / \sqrt{|a|}$, or $a<0$ and all the roots of Equation (2.2) with (3.2) are inside the disk $|\mu|<1 / \sqrt{|a|}$. We will first establish necessary and sufficient conditions for all the roots of Equation (2.1) with (3.2) (resp. Equation (2.2) with (3.2)) to be inside the disk $|\mu|<1 / \sqrt{|a|}$ in the case $a>0$ (resp. in the case $a<0$ ) and then complete the proof of Theorem 1.1.

Theorem 3.1. Let $c$ be nonzero real constant, a a positive constant and $k a$ positive integer. Then all the roots of Equation (2.1) are inside the disk $|\mu|<$ $1 / \sqrt{a}$, if and only if either of the following conditions holds:

(a) $k$ is odd, $0<a<1$ and $|c|<(1-a) / a^{(k+2) / 2}$;

(b) $k$ is even, $0<a<1 / \alpha^{2}$ and $(a-1) / a^{(k+2) / 2}<c<b_{*}\left(a, \phi_{0}\right)$, where $\phi_{0}$ is the one in Lemma 2.9.

Proof. Set $\delta(c):=\max \{|\mu|: \mu$ is a root of Equation (2.1)\}. Clearly, $\delta(c)$ is continuous in $c$ and $\lim _{|c| \rightarrow \infty} \delta(c)=\infty$. Consider the following two cases.

Case 1: $k$ is odd. By Lemma 2.1(a), $\delta(c) \geq 1$ for $c \in \boldsymbol{R}$, and hence a necessary condition for all the roots of Equation (2.1) to be inside the disk $|\mu|<1 / \sqrt{a}$ for some $c \in \boldsymbol{R}$ is $1<1 / \sqrt{a}$, that is,

$$
0<a<1 \text {. }
$$

Conversely, let us assume that (3.3) holds. Then it is obvious that $\delta(0)=$ $1<1 / \sqrt{a}$; and therefore by virtue of Remarks 2.1 and 2.2 , together with Lemma 2.6, one can see that all the roots of Equation (2.1) are inside the disk $|\mu|<1 / \sqrt{a}$ if and only if $-b_{*}(a, 0)<c<b_{*}(a, \pi)$, i.e., $|c|<(1-a) / a^{(k+2) / 2}$. Thus, we have Part (a) of the theorem.

Case 2: $k$ is even. By Lemma 2.1(b), Remark 2.1 and Lemma 2.6, we see that $\min _{c \geq 0} \delta(c)=\alpha$ and $\min _{c \leq 0} \delta(c)=1$. Consider the case $c \geq 0$. It follows from $\min _{c \geq 0} \delta(c)=\alpha$ that a necessary condition for all the roots of Equation (2.1) to be inside the disk $|\mu|<1 / \sqrt{a}$ for some $c \geq 0$ is $\alpha<1 / \sqrt{a}$, i.e., $0<a<1 / \alpha^{2}$. Now assume that $0<a<1$ holds. Then $\delta(0)=1<1 / \sqrt{a}$, and hence by Lemmas 2.1(b), 2.9 and 2.6, the supremum of $c$ such that all the roots of Equation (2.1) are inside the disk $|\mu|<1 / \sqrt{a}$ is $b_{*}\left(a, \phi_{0}\right)$. Therefore, in the case $0<a<1$, all the roots of Equation (2.1) are inside the disk $|\mu|<1 / \sqrt{a}$ if and only if

$$
0 \leq c<b_{*}\left(a, \phi_{0}\right) .
$$


Assume also that $1 \leq a<1 / \alpha^{2}$. By Lemma 2.1(b), Remarks 2.1 and 2.2, together with Lemma 2.9, the infimum and the supremum of $c$ such that all the roots of Equation (2.1) to be inside the disk $|\mu|<1 / \sqrt{a}$ are given by $b_{*}(a, 0)=(a-1) / a^{(k+2) / 2}$ and $b_{*}\left(a, \phi_{0}\right)$, respectively. By virtue of Lemmas 2.1(b) and 2.6 again, in the case $1 \leq a<1 / \alpha^{2}$, all the roots of Equation (2.1) to be inside the disk $|\mu|<1 / \sqrt{a}$ if and only if

$$
(a-1) / a^{(k+2) / 2}<c<b_{*}\left(a, \phi_{0}\right) .
$$

We next consider the case $c \leq 0$. One can then see from $\min _{c \leq 0} \delta(c)=1$ that a necessary condition for all the roots of Equation (2.1) to be inside the disk $|\mu|<1 / \sqrt{a}$ for some $c \leq 0$ is $1<1 / \sqrt{a}$, i.e., $0<a<1$. In view of Lemma 2.1(b), Remarks 2.1 and 2.2, together with Lemma 2.6, the infimum of $c$ such that all the roots of Equation (2.1) to be inside the disk $|\mu|<1 / \sqrt{a}$ is $(a-1) / a^{(k+2) / 2}$; and hence in the case $0<a<1$, all the roots of Equation (2.1) to be inside the disk $|\mu|<1 / \sqrt{a}$ if and only if

$$
(a-1) / a^{(k+2) / 2}<c \leq 0 .
$$

Consequently, it follows from (3.4), (3.5) and (3.6) that all the roots of Equation (2.1) to be inside the disk $|\mu|<1 / \sqrt{a}$ if and only if

$$
0<a<\frac{1}{\alpha^{2}} \quad \text { and } \quad(a-1) / a^{(k+2) / 2}<c<b_{*}\left(a, \phi_{0}\right),
$$

which yields Part (b) of the theorem.

The proof is complete.

Theorem 3.2. Let $c$ be nonzero real constant, a a negative constant and $k$ a positive integer. Then all the roots of Equation (2.2) are inside the disk $|\mu|<1 / \sqrt{|a|}$, if and only if either of the following conditions holds:

(a) $k$ is odd, $-1<a<0$, and $|c|<b_{*}\left(a, \phi_{1}\right)$, where $\phi_{1}$ is the one in Lemma 2.10 .

(b) $k$ is even, $-1 / \alpha^{2}<a<0$, and $(|a|-1) /|a|^{(k+2) / 2}<(-1)^{m} c<b_{*}\left(a, \phi_{2}\right)$, where $m=k / 2-2[k / 4]+1$ and $\phi_{2}$ is one in Lemma 2.10 .

Proof. Set $\delta(c):=\max \{|\mu|: \mu$ is a root of Equation (2.2)\}. Clearly, $\delta(c)$ is continuous in $c$ and $\lim _{|c| \rightarrow \infty} \delta(c)=\infty$. Consider the following two cases.

Case 1: $k$ is odd. By Lemma 2.7 and the fact $\delta(0)=1, \delta(c) \geq 1$ for $c \in \boldsymbol{R}$, and hence a necessary condition for all the roots of Equation (2.2) to be inside the disk $|\mu|<1 / \sqrt{|a|}$ for some $c \in \boldsymbol{R}$ is $1<1 / \sqrt{|a|}$, that is,

$$
-1<a<0
$$


Conversely, let us assume that (3.7) holds. By Lemmas 2.4(a), 2.7, Remark 2.2, together with Lemma 2.10(a), the infimum and the supremum of $c$ such that all the roots of Equation (2.2) to be inside the disk $|\mu|<1 / \sqrt{|a|}$ are given by $-b_{*}\left(a, \phi_{1}\right)$ and $b_{*}\left(a, \phi_{1}\right)$, respectively. Therefore, all the roots of Equation (2.2) are inside the disk $|\mu|<1 / \sqrt{|a|}$ if and only if

$$
-1<a<0 \quad \text { and } \quad|c|<b_{*}\left(a, \phi_{1}\right) .
$$

Thus, we have Part (a) of the theorem.

Case 2: $k$ is even. First assume that $k \equiv 2(\bmod 4)$. Notice that in this case, $m=k / 2-2[k / 4]+1$ is even. By Lemma 2.4(b), Remark 2.1 and Lemma 2.7, we see that $\min _{c \geq 0} \delta(c)=\alpha$ and $\min _{c \leq 0} \delta(c)=1$. Consider the case $c \geq 0$. It follows from $\min _{c \geq 0} \delta(c)=\alpha$ that a necessary condition for all the roots of Equation (2.2) to be inside the disk $|\mu|<1 / \sqrt{|a|}$ for some $c \geq 0$ is $\alpha<1 / \sqrt{|a|}$, i.e., $-1 / \alpha^{2}<a<0$. Now assume that $-1<a<0$ holds. Then $\delta(0)=1<$ $1 / \sqrt{|a|}$, and hence by Lemmas 2.4(b), 2.10(b) and 2.7, the supremum of $c$ such that all the roots of Equation (2.2) are inside the disk $|\mu|<1 / \sqrt{|a|}$ is $b_{*}\left(a, \phi_{2}\right)$. Therefore, in the case $-1<a<0$, all the roots of Equation (2.2) are inside the disk $|\mu|<1 / \sqrt{|a|}$ if and only if

$$
0 \leq c<b_{*}\left(a, \phi_{2}\right) .
$$

Assume also that $-1 / \alpha^{2}<a \leq-1$. By Lemma 2.4(b), Remarks 2.1 and 2.2, together with Lemma 2.10(b), the infimum and the supremum of $c$ such that all the roots of Equation (2.2) to be inside the disk $|\mu|<1 / \sqrt{|a|}$ are given by $b_{*}(a, \pi / 2)$ and $b_{*}\left(a, \phi_{2}\right)$, respectively. By virtue of Lemmas 2.4(b) and 2.7 again, in the case $-1 / \alpha^{2}<a \leq-1$, all the roots of Equation (2.2) to be inside the disk $|\mu|<1 / \sqrt{|a|}$ if and only if

$$
(|a|-1) /|a|^{(k+2) / 2}<c<b_{*}\left(a, \phi_{2}\right) .
$$

We next consider the case $c \leq 0$. One can then see from $\min _{c \leq 0} \delta(c)=1$ that a necessary condition for all the roots of Equation (2.2) to be inside the disk $|\mu|<1 / \sqrt{|a|}$ for some $c \leq 0$ is $1<1 / \sqrt{|a|}$, i.e., $-1<a<0$. In view of Lemma 2.4(b), Remarks 2.1 and 2.2, together with Lemma 2.7, the infimum of $c$ such that all the roots of Equation (2.2) to be inside the disk $|\mu|<1 / \sqrt{|a|}$ is $(|a|-1) /|a|^{(k+2) / 2}$; and hence in the case $-1<a<0$, all the roots of Equation (2.2) to be inside the disk $|\mu|<1 / \sqrt{|a|}$ if and only if

$$
(|a|-1) /|a|^{(k+2) / 2}<c \leq 0 .
$$

Consequently, it follows from (3.8), (3.9) and (3.10) that all the roots of Equation (2.2) to be inside the disk $|\mu|<1 / \sqrt{|a|}$ if and only if

$$
-1 / \alpha^{2}<a<0 \quad \text { and } \quad(|a|-1) /|a|^{(k+2) / 2}<c<b_{*}\left(a, \phi_{2}\right) .
$$


We next assume that $k \equiv 0(\bmod 4)$. Notice that in this case, $m=k / 2-$ $2[k / 4]+1$ is odd. By Lemma 2.4(c), Remark 2.1 and Lemma 2.7, we see that $\min _{c \leq 0} \delta(c)=\alpha$ and $\min _{c \geq 0} \delta(c)=1$. Similarly, we can obtain that a necessary condition for all the roots of Equation (2.2) to be inside the disk $|\mu|<1 / \sqrt{|a|}$ for some $c \leq 0$ is $-1 / \alpha^{2}<a<0$; and furthermore, By Lemma 2.4(c), Remarks 2.1 and 2.2, together with Lemmas 2.7 and 2.10(b), that all the roots of Equation (2.2) to be inside the disk $|\mu|<1 / \sqrt{|a|}$ if and only if

$$
-1 / \alpha^{2}<a<0 \quad \text { and } \quad-b_{*}\left(a, \phi_{2}\right)<c<-(|a|-1) /|a|^{(k+2) / 2} \text {. }
$$

Combining (3.11) and (3.12), we have Part (b) of the theorem.

The proof is complete.

Combining Theorem 3.1 with Theorem 3.2 and taking (3.2) into account, we complete the proof of Theorem 1.1.

Acknowledgement. The author is grateful to the Referee for his appropriate comments and careful review work on the present paper.

\title{
References
}

[1] Kuruklis, S. A., The asymptotic stability of $x_{n+1}-a x_{n}+b x_{n-k}=0$, J. Math. Anal. Appl., 188 (1994), 719-731.

[2] Dannan, F. M., The asymptotic stability of $x(n+k)+a x(n)+b x(n-l)=0$, Journal of Difference Equations and Applications, 10:6 (2004), 589-599.

\author{
nuna adreso: \\ Institute of Mathematics Science \\ Heilongjiang University \\ Harbin, 150080 \\ P. R. China \\ E-mail: renhsh150080@163.net
}

(Ricevita la 12-an de junio, 2006)

(Reviziita la 15-an de aprilo, 2007) 